des échantillons et nous avons obtenu la moyenne la plus petite de 0 mgr. 75 par litre de lait de vache ou 0 mgr. $075 \%$.

La méthode de Folin, Burstein et From, simple dans son principe, est dans l'exécution assez compliquée surtout en la modification de Kluge; au point de vue de la vitesse, de la facilité de l'exécution, de la précision basée sur le système des contrôles parfaits, elle n'est pas à comparer avec la méthode de J. K. Parnas.

\title{
Conclusions
}

1. Le but de notre étude était de déterminer la quantité d'ammoniaque dans le lait frais de vache obtenu avee l'exclusion parfaite des facteurs extérieurs.

2. Dans les 43 échantillons, la quantité d'ammoniaque oscillait dans les limites de 0 à $2 \mathrm{mgr}$. 18 par litre, et la quantité moyenne était de 0 mgr. 75 par litre ou 0 mgr. $075 \%$; le taux d'ammoniaque dans le lait de quartiers séparés de la même vache et de la même traite est soumis à des oseillations sensibles, ainsi que dans le lait de différentes vaches.

3. Etant donné que l'augmentation du taux d'ammoniaque dans le lait dépend de la déeomposition des substanees protéiques du lait par les bactéries protéolysantes pendant sa conservation, l'initiative reprise de l'application du dosage de l'ammoniaque dans le lait pour l'estimation de sa valeur hygiénique, particulièrement du lait de première qualité pour les nourrissons, du lait pasteurisé, stérilisé, condensé, mérite d'être un sujet d'études consécutives, qui sont en cours dans notre Institut.

4. Parmi les méthodes du dosage de l'ammoniaque dans le lait, la méthode de J. K. Parnas est à choisir de préférence.

\section{LA FENAISON ARTIFICIELLE}

\section{par \\ G. GÉNIN}

Il y a environ une dizaine d'années, un spéeialiste de l'Eniversité de Cambridge avait constaté que l'herbe jeune possédait une valeur nutritive beaucoup plus élevée pour le bétail que l'herbe venue à maturité. Il avait constaté en outre que cette herbe ne perd presque aucune de ses propriétés par séchage artificiel.

Depuis ces observations, on s'est appliqué à rechercher un procédé permettant de produire du foin artificiel, e'est-à-dire du foin séché par un procédé artificiel, à un prix pouvant concurrencer celui des tourteaux. Depuis quelques mois, les démonstrations se multiplient en Angleterre d'un nouveau procédé de séchage qui a donné 
tous les résultats escomptés et qui permet d'obtenir, au prix de 4 livres par tonne, une herbe séchée, alors que tous les autres aliments employés pour la nourriture des animaux reviennent à une moyenne de 7 livres. La ferme où cette démonstration a été faite, qui se trouve à Stratford-on-Avon et qui appartient à un groupe de fermes modèles, a d'ailleurs décidé d'abandonner la fenaison naturelle pour avoir recours à ce nouveau procédé de séchage de l'herbe.

Voici d'ailleurs un résumé d'un compte rendu publié à la suite d'essais de séchage artificiel, qui eurent lieu les 28 et 29 avril dernier. La ferme dans laquelle ces essais furent effectués, qui porte le nom de Spring Farm, appartient à un groupe de trois fermes voisines qui ont à leur disposition une superficie de 280 hectares environ. Jusqu'à ces dernières années, les travaux dans ces fermes étaient très divers : prairies, culture de l'orge, des betteraves, navets, choux raves, etc. Aujourd'hui au contraire toutes les terres ont été tansformées en prairies et les seuls produits des fermes sont le lait et le foin.

Le sol qui constitue les terres de ces exploitations agricoles est de qualité très variable: sable, gravier, argile, etc. A la Spring Farm, on s'occupe principalement de l'élevage du jeune bétail ; au contraire à la Ferme d'Hatton Rock, la plus voisine, on élève le troupeau de vaches laitières, enfin à la Blacon Farm se trouve le troupeau de vaches ne produisant pas de lait et de génisses. C'est à la Blacon Farm que se trouve le second appareil de séchage de l'herbe, le premier étant à la Spring Farm. Le troupeau se compose de 370 vaches d'Ayrshire de race reconnue et soigneusement contrôlée, dont 120 sont en période de lactation.

\section{Essai de séchage en 1935.}

En 1935, l'herbe récoltée sur le territoire des fermes de Hatton Rock et Spring Farm, avait été séchée dans un petit appareil d'essai. Le rendement, après une seule coupe, avait été d'environ 2 tonnes d'herbe sèche par hectare, mais il avait été possible dans certains champs d'effectuer une seconde et même une troisième et quatrième récolte. C'est le 25 avril que les premiers essais de séchage artificiel furent effectués, ils se terminèrent le 16 octobre de la même année.

200 tonnes d'herbe sèche furent ainsi préparées et la plus grande partie de cette herbe aurait été perdue en l'absence d'un appareil sécheur. Le prix de cette herbe sèche s'éleva à 4 livres par tonne et cet aliment fut donné aux animaux à la place de tourteaux divers dont le prix atteignait 7 livres par tonne. Cette herbe qui renfermait 15 à $18 \%$ de protéines après séchage fut distribuée aux 'animaux dans la proportion de 8 pounds pour 8 livres de lait données par l'animal. On constata que pendant tout l'hiver, le lait conservait 


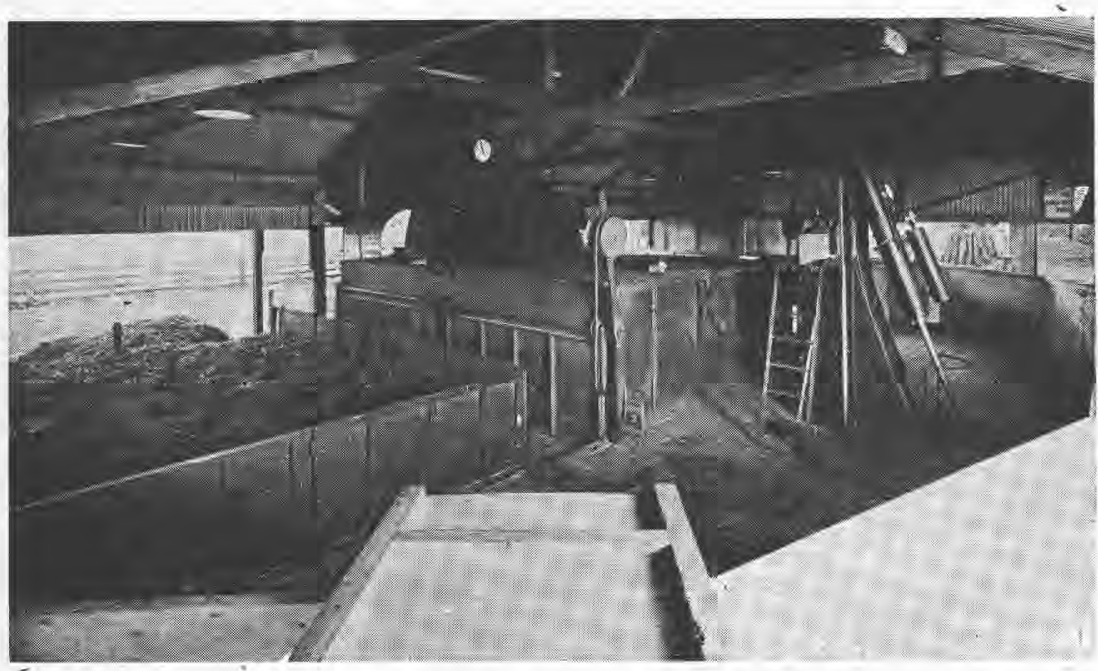

Sécheur d'herbe de Billigam

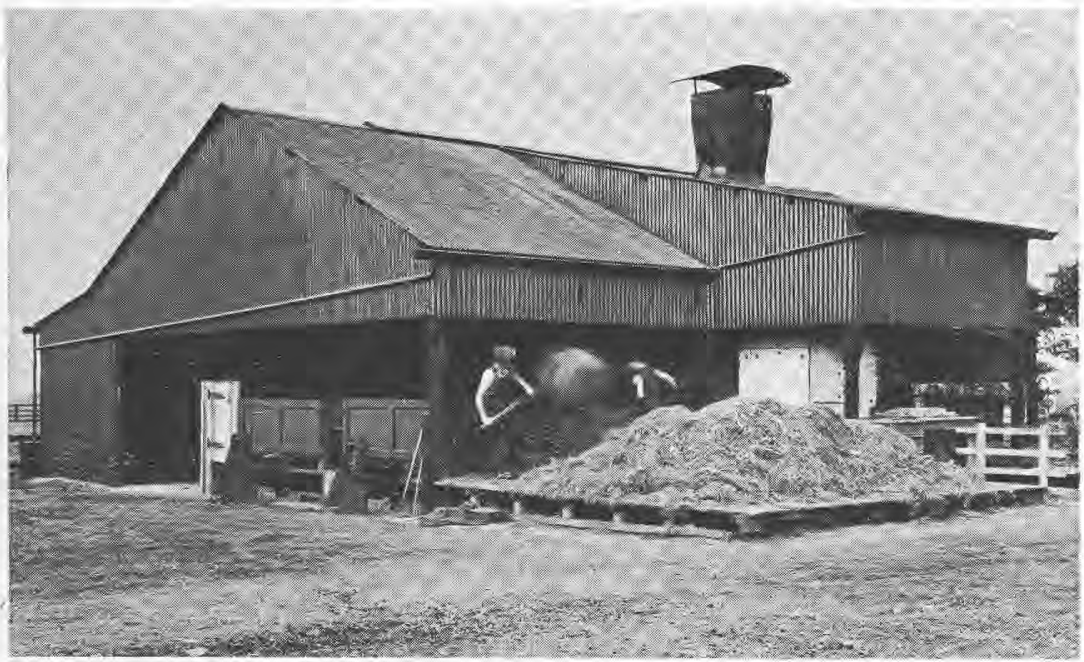

Mise en place de l'herbe fraîchement coupée dans les compartiments de séchage de l'appareil

les caractéristiques qu'il possède lorsque l'animal se trouve dans les pâturages pendant les mois d'été.

Une herbe sèche, mais de qualité un peu inférieure, fut donnée aux veaux et on constata que la croissance des jeunes animaux était 
au moins aussi bonne que celle de veaux recevant d'autres genres de nourriture.

\section{Essais de séchage en 1936.}

En 1936, les expériences furent poursuivies sur 2 sécheurs de dimensions beaucoup plus grandes, prévus en vue de traiter l'herbe fournie par une exploitation d'environ 160 hectares. L'une de ces installations se trouvait à la Spring Farm, l'autre à la Blacon Farm. Les frais d'installation de cet appareil s'étaient élevés pour chacun d'eux, aux sommes suivantes:

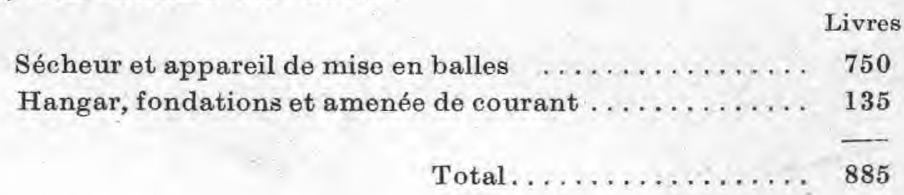

La conduite du sécheur exige la présence de deux hommes et celle de l'appareil de mise en balles la présence d'un homme. Chaque sécheur fonctionnait en coopération avec un tracteur, une moissonneuse et un distributeur d'engrais.

On peut estimer que la quantité d'herbe sèche produite pendant 8 heures s'élève à une moyenne de 2 tonnes d'herbe, mais ce rendement varie avec la quantité d'eau contenue dans l'herbe fraîchement coupée et il augmente vers la fin de la saison. Les premières récoltes effectuées au début d'avril, donnent un rendement d'environ $350 \mathrm{~kg}$. d'herbe sèche à l'acre (0 hectare 40$)$; au contraire aveo les dernières coupes, le rendement peut s'élever jusqu'à 1.250 kilogrammes. En moyenne, on peut admettre qu'il atteint 2 tonnes par hectare.

L'analyse de l'herbe séchée en avril a donné les résultats suivants :

\begin{tabular}{|c|c|}
\hline Equivalent de protéines & 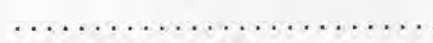 \\
\hline quivalent d'amidon ... & $\ldots \ldots \ldots \ldots \ldots \ldots$, \\
\hline
\end{tabular}

L'excellente couleur de cette herbe sèche montre qu'elle est riche en carotène, matière initiale, rappelons-le, de la vitamine $\mathrm{A}$ ou vitamine de la croissance.

Ce nouveau procédé de fenaison artificielle permet donc de donner aux animaux, d'un bout de l'année à l'autre, du foin d'excellente qualité, et d'augmenter considérablement le rendement des prairies. Cette nourriture donne aux animaux la totalité des substances dont ils ont besoin, de sorte qu'il est inutile d'ajouter à ce foin des tourteaux ou autres substances. Si au point de vue volume, on estime 
que l'herbe séchée artificiellement représente un volume insuffisant, on peut la mélanger à une herbe de moins bonne qualité.

Dans les fermes qui nous occupent, la quantité d'herbe séchée récoltée sur l'ensemble des terrains, était supérieure à celle nécessaire à la nourriture du troupeau, le surplus était donc vendu.

\section{Dépenses de séchage.}

Voici comment s'établit le prix de revient d'une tonne d'herbe sèche :

L. s. d.

Main-d'œuvre (séchage et mise en balles)..

Coke .............................

Electricité.

Feuillards pour la mise en balles.

Frais d'entretien et réparations

Récolte et transport de l'herbe fraîche

Amortissement du matériel et intérèt du eapital mobilisé

Total
130

16

16

56

2

120

$\begin{array}{ll}7 & 6\end{array}$

Mais il ne faut pas oublier que ce procédé permet d'utiliser l'herbe récoltée pendant toute la durée de la saison, alors qu'autrement, une grande partie de cette herbe serait perdue par suite du temps non favorable pour la fenaison.

\section{Emploi des engrais.}

Il importe, pour obtenir un rendement maximum en herbe fraîche, d'utiliser les engrais appropriés pour activer la croissance de l'herbe. L'utilisation des engrais commence vers la mi-février et on répand sur les prairies une quantité d'engrais concentrés complets correspondant à environ 200 kilogrammes par hectare. Cette application n'est pas faite au même moment sur toutes les prairies, mais on la décale dans le temps jusqu'à fin mars, afin que l'herbe ne soit pas prête à être coupée dans tous les champs au même moment, mais qu'il y ait également un décalage dans la récolte.

On fait une seconde application d'engrais complet ou de nitrochalk suivant les circonstances après la première ou la seconde coupe d'herbe. L'emploi du fumier est réservé à l'automne et il est également préférable d'utiliser une quantité importante d'engrais de cette sorte tous les 3 ou 4 ans.

\section{Détail de construction du sécheur.}

Si nous considérons une installation normale, elle se compose au centre de l'installation d'un four et de chaque côté d'une aire sur laquelle on vient livrer l'herbe destinée à être séchée. Ce four brûle 
du coke et il fonctionne d'une façon continue; il suffit chaque quinzaine de l'arrêter, en vue d'éliminer les crasses et scories. Au moyen de ce four, on produit un gaz qui est brûlé dans 6 brûleurs placés à la partie supérieure de l'installation. Les gaz de combustion chauffent le courant d'air dirigé dans l'appareil et le portent à une température de $150^{\circ}$. Cet air passe derrière le four et arrive à la partie inférieure du sécheur.

On place dans ce sécheur deux plateaux contenant l'herbe à sécher. L'air passe sur ces plateaux en entraînant l'humidité contenue dans l'herbe et l'air humidifié s'échappe par une cheminée dans l'atmosphère. La circulation de l'air du four vers le sécheur est assurée par un ventilateur commandé au moyen d'un moteur électrique, cet ensemble étant placé à la partie supérieure de la chambre de séchage. Cette dernière porte deux portes sur chaque face opposée, de sorte que lorsqu'on introduit deux plateaux chargés d'herbe humide par un côté, on peut enlever les plateaux d'herbe sèche par un autre côté. On voit donc qu'en ayant 4 plateaux, il y en a toujours une paire dans la chambre de séchage, tandis que l'autre paire est en période de chargement ou de déchargement:

Chaque chargement d'herbe fraîche fait un double séjour dans la chambre de séchage et occupe dans cette ehambre deux positions successives. Lorsque l'herbe est tout à fait humide, elle est placée dans la partie de la chambre où se fait l'entrée des gaz ayant déjà travaillé. Lorsque le chargement d'herbe a été partiellement séché, on le place dans une seconde position où s'effectue le traitement final, avec les gaz les plus secs et les plus chauds et cette opération en contre-courant donne les résultats les meilleurs. C'est le thermomètre placé à l'intérieur de la cheminée qui indique lorsque l'herbe est sèche ; l'expérience en effet a montré qu'on peut estimer que toute l'humidité a été chassée, lorsque la température des gaz sortant de l'appareil atteint $75^{\circ} \mathrm{C}$. L'herbe sèche est alors comprimée en balles d'environ 40 kilogrammes.

Cette question du séchage de l'herbe a été suivie par plusieurs personnalités éminentes et des communications du plus grand intérêt ont déjà été présentées devant diverses Commissions techniques et scientifiques.

Les Professeurs J. E. S. Watson, R. G. Stapledon et S. J. WATSON ont en particulier publié plusieurs études auxquelles nous avons emprunté quelques-uns des renseignements qui suivent.

D'après le Professeur J. E. S. Watson, le procédé de séchage artificiel conserve la totalité de la valeur alimentaire de l'herbe fraîche. Il n'y a pas perte de matière sèche, pas diminution de la digestibilité, ni diminution de la proportion de vitamine. Pour donner un chiffre, on peut dire qu'une tonne d'herbe séchée artificielle- 
ment présente 2 fois la valeur nutritive d'une tonne de foin de qualité moyenne. Etant donné qu'avec le climat anglais, on peut prévoir, si le terrain reçoit une quantité suffisante d'engrais, une récolte assez importante d'herbe, on voit quel est l'avantage économique du séchage artificiel.

Certaines précautions doivent être prises au cours de l'opération; il faut en particulier, pour éviter un grillage du produit, une certaine pratique de la conduite de l'opération. De même, on doit s'efforcer de réaliser des économies de combustible et lorsque le temps le permet, il y a avantage à laisser sécher pendant peu de temps l'herbe sur le sol. Si l'herbe est en effet traitée, alors qu'elle est encore recouverte de rosée, il faut éliminer 5 tonnes d'eau pour obtenir une tonne d'herbe sèche, d'où diminution du rendemént de l'opération et augmentation de la dépense de combustible. Si au contraire la rosée a été éliminée par un séchage préalable, la quantité d'eau à éliminer n'est plus que de 3 tonnes, si enfin on prolonge le séchage pendant 48 heures, il n'y a plus que 1 tonne 25 d'eau à éliminer. Evidemment dans ce cas, il y a une légère perte de carotène, mais le produit initial en contient une quantité largement suffisante pour que l'herbe séchée constitue encore une excellente nourriture d'hiver.

Signalons en passant qu'on annonce un nouveau type de sécheur dans lequel l'herbe serait comprimée entre des rouleaux. Le résidu solide provenant de ce traitement serait séché et le jus serait évaporé et donnerait un produit auquel on a donné le nom de crème d'herbe à très haute teneur en vitamine.

M. S. J. WAtson a principalement examiné la question de la nourriture des animaux par l'herbe sèche. Il a tout d'abord constaté que cette herbe renferme tous les produits nutritifs de l'herbe initiale, ainsi qu'on peut s'en assurer par détermination de l'amidon, de protéines digestibles, etc. Cette herbe sèche contient également des produits minéraux sous une forme assimilable et ceci est très important, car on a constaté par exemple que la fièvre de lait est souvent due à une déficience du calcium dans le sang. Par séchage artificiel, on maintient done la teneur en phosphore et en ealcium du produit à une valeur optimum. De même, le fer est également conservé, ainsi que la vitamine $\mathrm{A}$.

En ce qui concerne plus particulièrement la nourriture des vaches laitières, l'emploi d'herbe sèche augmente sensiblement la teneur en résidu sec du lait. Celui-ci conserve en outre l'hiver la coloration jaune qu'il avait l'été. L'emploi d'herbe sèche constitue également un moyen pour accélérer la croissance des jeunes animaux et accélérer leur engraissement. Enfin, les mêmes avantages se retrouvent pour la nourriture des moutons, des porcs, de la volaille et des chevaux. 